\title{
Shape Grammar applied to urban morphology studies: land subdivision in urbanized areas.
}

\author{
SIGRADI2018 \\ TECHNOPOLITICAS \\ xxii congresso da sociedade \\ iberoamericana de gráfica digita \\ 22th conference of the \\ iberoamerican society \\ of digital graphics \\ 07|08|09|novembro|2018 \\ iau usp | são carlos | sp br \\ Moacir Cassiano \\ UFPB | Brazil | moacircassiano@ct.ufpb.br \\ Lilian L. Félix \\ UFPB | Brazil | lilianlfelix@gmail.com \\ Cristiana Griz \\ UFPE | Brazil | crisgriz@gmail.com
}

\begin{abstract}
Currently, the land regulation plans define the urban micro-scale, producing standardized and pre-dimensioned blocks and lots, generating monotony, poor urban quality and high infrastructure costs. In the quest for sustainability, studies point to a density and a certain degree of population density to enable urban infrastructures with qualitative and quantitative gains, through concepts of compactness, completeness and connectivity. This study presents possibilities of batch sizing using the Grammatical method of the Form, through morphological configurations and rules presents possibilities of generation of new formats, distribution of urban lots in the neighborhood Valentina, in João Pessoa - PB and discusses results.
\end{abstract}

Keywords: Urban Lots; Shape Grammar; Sustainability; Urban Density;

\section{INTRODUÇÃO}

Atualmente o parcelamento do solo na microescala urbana é definido por regras que se baseiam nos planos e políticas de regulamentação do solo, produzindo quadras e lotes padronizados e pré-dimensionados (ROLNIK et al, 2011). Essa padronização favorece a produção de um cenário monótono, de pouca qualidade urbana na escala de vizinhança e de altos custos com infraestrutura (JACOBS, 2000)

Estudos apontam que os custos com infraestrutura são os de maior impacto na área urbana e argumentam que dependendo do grau de densidade populacional em determinadas áreas é possível obter ganhos expressivos quantitativos e qualitativos, promovendo a sustentabilidade urbana através de conceitos como compacidade, completude e conectividade (PONT \& HAUPT, 2010; GAUZIN-MÜLLER, 2002, FARR, 2013; GEHL, 2013; SILVA et al 2016).

Neste processo de busca pela sustentabilidade, o adensamento é um dos fatores preponderantes e a forma edificada assume papel fundamental. No entanto, a edificação normalmente é limitada por diretrizes projetuais de leis de zoneamento e uso do solo que se embasam no dimensionamento do lote em que será implantada. Por meio de novas possibilidades de dimensionamento de lotes, questões como a verticalização e a distribuição espacial das edificações se apropriariam de maior maleabilidade e performance, favorecendo a criação de novos cenários com maior variedade e promovendo qualidade urbana.

O processo experimental do uso do método da gramática da forma, no campo do urbanismo sustentável, mostra-se pertinente em estudos para definição de lotes urbanos, abordando aspectos de configuração do espaço como forma e densidade. $\mathrm{Na}$ busca por estratégias para projetos urbanos sustentáveis, a gramática da forma (STINY, GIPS, 1971) apresenta possibilidades de criar formatos e distribuição de lotes, pois é um sistema generativo que consiste em um sistema que desenvolve formas baseado em regras que são aplicadas passo a passo, para gerar uma linguagem de novos projetos que atendam a requisitos pré-estabelecidos.

Este artigo apresenta a aplicação da Gramática da Forma para definição de lotes urbanos em estudos sobre urbanismo sustentável no departamento de PósGraduação em Arquitetura e Urbanismo da Universidade Federal da Paraíba em 2017. Os resultados são obtidos por meio de três derivações a partir de um corpus selecionado e a discussão aponta como estes resultados contribuem para projetos urbanos sustentáveis. 


\section{REVISÃO TEÓRICA}

Sobre as origens da Gramática da Forma (shape gramar), Celani (2002) apresenta sua relação com o sistema de produção do matemático Emil Post (1943) e com a gramática generativa do linguista Noam Chomsky (1957) e explica que ela "consiste em um sistema de geração de formas baseado em regras" desenvolvido por George Stiny e James Gips na década de 70. Além das regras, são inferidas restrições para o desenvolvimento de formas e seu formalismo se dá por destacar características geométricas, ressaltando a forma por meio de derivações (CELANI at. al, 2006).

Em 2001, Thomas Fischer e Christiane M. Herr apresentaram a importância do design generativo no meio acadêmico. Eles mostraram "experiências em ensino de design generativo, com foco na relação entre reprodução algorítmica da natureza e inovação" (FISCHER, HERR, 2001), esclarecem que o design generativo "consiste em uma metodologia projetual que tem foco no processo de projeto do qual decorre o produto que está sendo projetado". Assim a crescente aplicação da Gramática da Forma se consolidava também na área do desing.

Segundo Celani at. al (2006), pouco se discutia e não existia uma bibliografia organizada sobre o assunto. Descreve que inicialmente "o objetivo da gramática da forma era servir de sistema de geração de formas para a pintura e escultura". Os artistas pensavam em formas geométricas e formulavam regras que combinavam as formas de diversos modos, gerando uma obra de arte.

Desde então, o sistema evoluiu e novas características foram acrescentadas possibilitando novas aplicações. Celani at. al (2006) cita pelo menos cinco tipos diferentes de variações de gramáticas:

"Gramática da Forma Analítica: a primeira aplicação analítica foi proposta por Stiny (1977), para a caracterização de um conjunto de regras capaz de gerar desenhos de janelas tradicionais chinesas. Trata-se de um tipo de composição muito simples, baseado na subdivisão sucessiva de retângulos. Após esse trabalho, passaram a predominar as aplicações analíticas da gramática da forma;

Gramática Paramétrica: as primeiras definições de gramática da forma já sugeriam a possibilidade de que certos valores fossem deixados em aberto para serem definidos no momento da implementação. Dessa forma, é possível definir uma gramática da forma paramétrica, capaz de gerar uma enorme variedade de resultados;

Gramática Predefinida: (set grammar): trata-se de um tipo de gramática determinística em que uma mesma regra ou uma mesma sequência de regras é aplicada sucessivamente. Os resultados obtidos com esse tipo de gramática apresentam sempre alguma regularidade. Esse conceito foi aplicado, em especial, no artigo em que Stiny introduz a gramática da forma tridimensional utilizando os blocos de Froebl (STINY, 1980);

Gramática com Marcadores: o uso de marcadores (labels), que nada mais são que marcas aplicadas às formas para reduzir sua ordem de simetria, restringe a maneira como as regras podem ser aplicadas, mas, por outro lado, permitem maior controle sobre os resultados;
Gramática da Cor: desenvolvida por Knight (1993, 1994), essa variação da gramática da forma utiliza cores no lugar de marcadores. A maior vantagem é que elas podem ser utilizadas para a aplicação posterior de "regras de decoração", nas quais elementos extras podem ser inseridos nas áreas coloridas."

O uso da Gramática da Forma vem sendo amplamente difundido entre pesquisadores em arquitetura e urbanismo (FISCHER, HERR, 2001; DUARTE, 2005; BEIRÃO, 2015; CELANI at. al, 2006), em função do crescente reconhecimento da importância da metodologia deste sistema analítico e generativo nos processos criativos.

Para propor o desenvolvimento de uma gramática a partir de um conjunto de projetos existentes, Duarte se apropria da lógica da gramática para gerar novas soluções com base nos projetos de Álvaro Siza no conjunto da Malagueira, mostrando que novos projetos surgiram por meio da aplicação de diferentes regras gramaticais (DUARTE, 2005).

Em 2014, Beirão at. al. (2015) apresentou os resultados de um workshop realizado no curso de Arquitetura e Urbanismo da Faculdade de Engenharia Civil, Arquitetura e Urbanismo, da Universidade Estadual de Campinas. Para o workshop, utilizaram um "método de projeto definido como CityMaker, que foi desenvolvido por Duarte e Beirão (2012) e consiste em um método associado a um conjunto de ferramentas para gerar soluções alternativas para um contexto urbano. O método propõe a utilização de um conjunto combinado de padrões de projeto (design patterns) que codificam ações típicas de projeto usados por urbanistas. Dentre os procedimentos metodológicos empregados no workshop destaca-se a aplicação da Gramática da Forma. Beirão at. Al. (2015), defendem que as "estratégias baseadas em regras consiste em definir situações em que determinado elemento pode ser conectado a outro, e de que maneira isto pode ocorrer da forma para gerar lotes urbanos a partir de um corpus tradicional de um conjunto de quadras" destacando a aplicação da Gramática da Forma, combinando regras com a variação paramétrica para gerar uma grande quantidade de resultados

Este artigo apresenta a abordagem da Gramática da Forma como sistema generativo para definição de lotes urbanos em um conjunto de quadras selecionadas (corpus) no bairro Valentina em João Pessoa - PB. Tratase da aplicação prática em estudos para pesquisas no campo do urbanismo em desenvolvimento no departamento de Pós-Graduação em Arquitetura e Urbanismo da Universidade Federal da Paraíba em 2017.

\section{MÉTODO E PROCEDIMENTOS}

O método analítico adotado é quanti-qualitativo e caracteriza-se por quantificar dados e assimilar e/ou interpretar os mesmos dentro de uma abordagem conceitual (RICHARDSON, 2017). Neste artigo, esse método é identificado ao aplicar os conceitos de uma metodologia projetual já difundida e ao produzir um quantitativo de resultados proveniente da aplicação de diretrizes projetuais em uma operação lógica.

A metodologia projetual consiste na aplicação de estratégias compositivas baseadas no formalismo da 
gramática da forma. Como comentado, ela consiste em um processo generativo, no qual o foco principal é o processo de criação do projeto.

A gramática da forma é o tipo analítica, ao realizar a análise de um corpus (parcela da malha urbana) e identificar as características do mesmo, como o vocabulário da forma. Em seguida, ela passa a ser do tipo original, (segundo Duarte,2007), ao gerar novos projetos que atenderam a requisitos pré-estabelecidos, baseados em regras, as quais são aplicadas passo a passo. (STINY, GIPS, 2006; ELOY, 2012).

O procedimento realizado para a aplicar o formalismo da gramática da forma partiu da escolha de um corpus de análise. Neste caso, o corpus consiste em um parcelamento local, situado no Bairro de Valentina, em João Pessoa - PB, para que a gramática fosse capaz de reproduzir a mesma linguagem. Por meio da análise desse corpus é identificado o vocabulário de formas e são criadas e inferidas as regras da gramática. Essas regras, por sua vez, são aplicadas passo a passo, constituindo a derivação e gerando novos parcelamentos (ver figura 1).

As etapas do procedimento descrito anteriormente podem ser descriminadas da seguinte forma:

a. escolha de um corpus de análise - identificação do vocabulário de formas;

b. criação e inferência de regras;

c. produção das derivações - averiguação a capacidade de gerar novos parcelamentos com a mesma linguagem do corpus;

Por fim, seguiu-se com a proposição de cenários de massas edificadas inseridas nos novos parcelamentos. A simulação teve enfoque na visualização de espaços mais densificados, com variação na forma tanto da massa edificada como dos lotes.

\section{OBJETO DE ESTUDO E PROCESSO DE ANÁLISE}

O objeto de estudo caracteriza o corpus de análise: um conjunto de quadras em uma área urbana no bairro Planalto da Boa Esperança, em João Pessoa - PB. As quadras apresentam formato retangular e diferenciam-se entre si pela variedade de tamanho dos lotes e, consequentemente, das próprias quadras (ver figura 2).

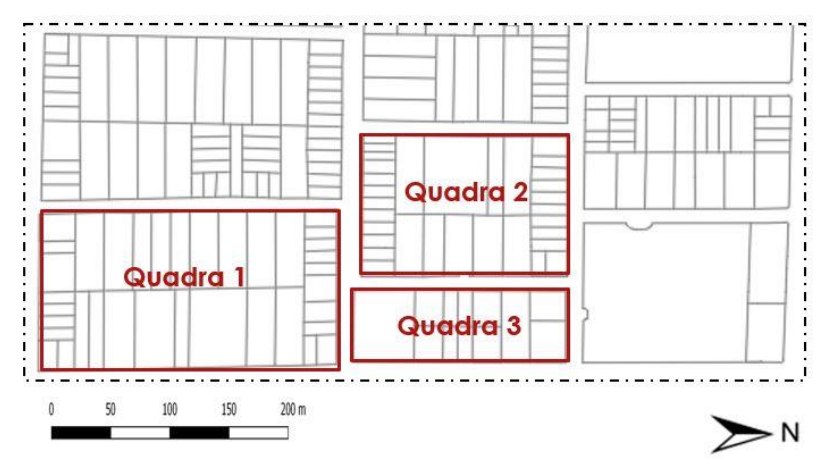

Figura 2: Corpus de análise, com destaque das quadras 1, 2 e 3, escolhidas para inferência das regras. Fonte: Autores (2018).

O vocabulário, definido na fase inicial do processo de criação da gramática da forma é o retângulo, visto que é determinado pela forma primitiva que compõe o corpus de análise, como ilustra a figura 3.

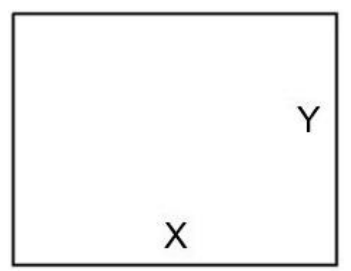

Figura 3: Vocabulário da gramática da forma. Fonte: Autores (2018)

Em seguida, para criar possíveis arranjos espaciais de lotes nas quadras, foram definidas regras e restrições para o desenvolvimento de uma gramática generativa, em um processo do tipo top-down que parte da subdivisão
3

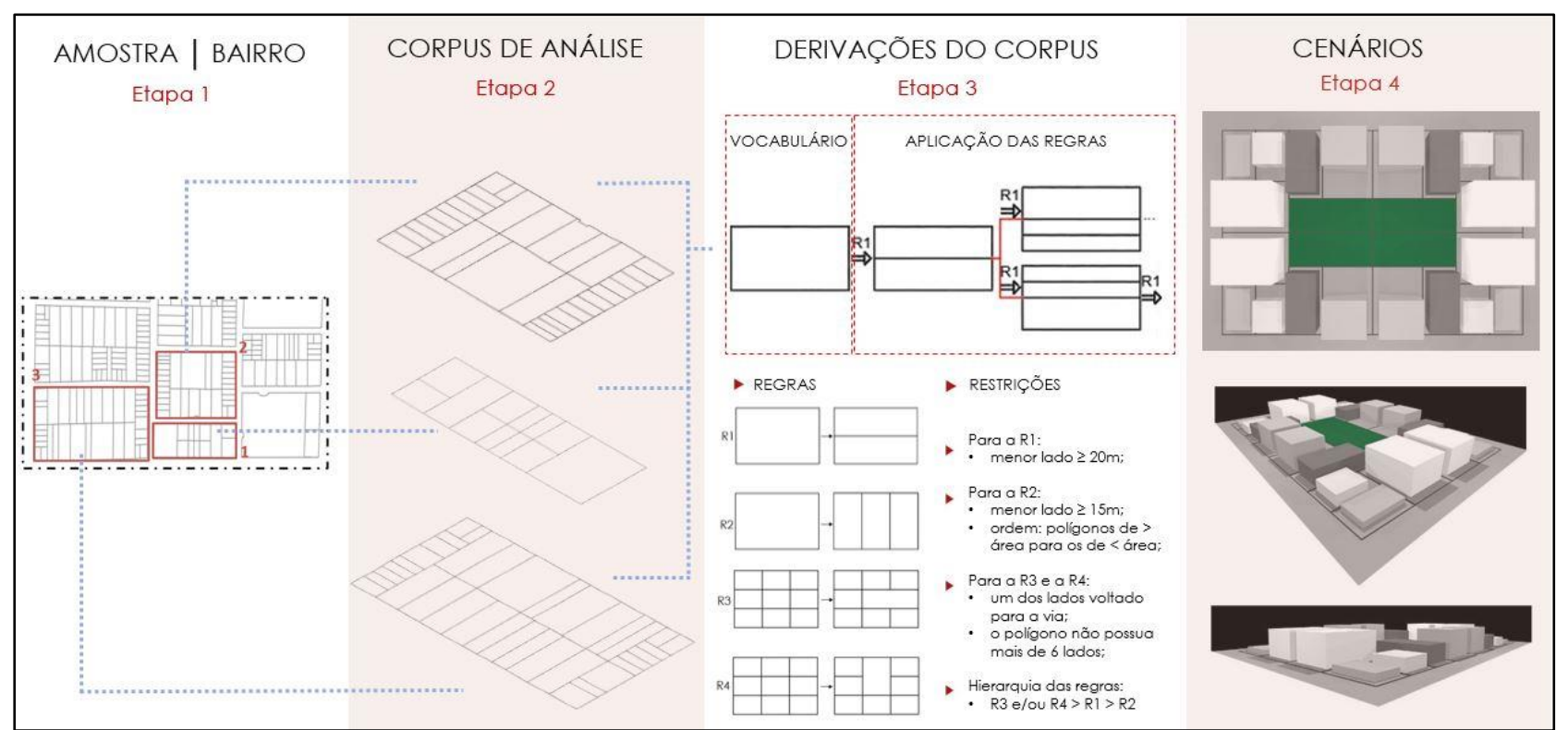

Figura 1: Diagrama do processo metodológico.

Fonte: Autores (2018). 
em sequência de uma forma maior.

As regras inferidas tiveram como partido a análise de especificações referentes ao dimensionamento dos lotes no código de urbanismo vigente, como dimensão da testada dos lotes, recuos em geral, taxa de ocupação e gabarito das edificações. Essas especificações foram escolhidas por influenciam diretamente na capacidade de densificação de uma área e, portanto, na possibilidade de aumentar a compacidade do local, promovendo a sustentabilidade urbana. Assim, as regras criadas para gerar o rearranjo espacial e redimensionamento dos lotes são (ver figura 4):

- R1: Divide-se o retângulo ao meio, paralelo ao lado de maior comprimento, desde que o menor lado, resultante da divisão, não fique com menos de $20 \mathrm{~m}$;

- R2: Divide-se o retângulo em três partes iguais, paralelo ao menor comprimento, desde que o menor lado, resultante da divisão, não fique com menos de $15 \mathrm{~m}$;

- R3 e R4: Sempre que um retângulo estiver enclausurado nos quatro lados, deve-se subtrair uma das faces, de modo que o novo polígono passe a ter pelo menos uma das faces livre e não possua mais de seis faces;

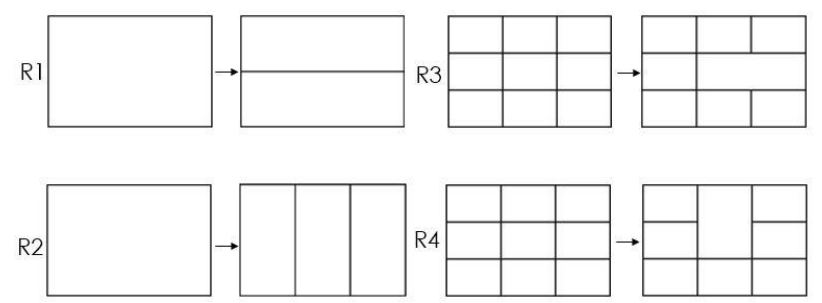

Figura 4: Ilustração das regras aplicadas no processo de derivação da gramática da forma. Fonte: Autores (2018)

Durante o processo de desenvolvimento, houve a necessidade de inserir algumas restrições que garantissem a aplicabilidade das regras, tais como:

- Para aplicar a regra R1, a dimensão do menor lado do polígono selecionado deve ser maior ou igual a $20 \mathrm{~m}$;

- Para aplicar a regra R2, a dimensão do menor lado do polígono selecionado deve ser maior ou igual a $15 \mathrm{~m}$. A regra deve ser aplicada na sequência dos polígonos de maior área para os de menor área;

- Para aplicar as regras R3 e R4, o polígono selecionado deve resultar com um dos lados voltado para o exterior da quadra (no caso, para a via). O polígono final não pode ter mais de seis lados.

- $\quad$ As regras devem ser aplicadas na seguinte hierarquia: $\mathrm{R} 3$ e $\mathrm{R} 4>\mathrm{R} 1>\mathrm{R} 2$

Após a definição das regras e restrições são realizadas as derivações gerando diversos resultados de lotes redimensionados e com arranjos espaciais variados nas quadras selecionadas. O processo foi realizado para cada uma das três quadras devido a variação de tamanho que elas apresentam entre si, como forma de verificar a aplicabilidade das regras para qualquer dimensão de quadra. A figura 5 ilustra a derivação da quadra 1, sobre a qual foram realizadas as simulações de cenários com edificações.

O limite de aplicabilidade da gramática torna-se evidente quando não é mais possível a aplicação das regras. Nesse caso, o que determina esse limite é a dimensão das faces que deve obedecer a um tamanho mínimo, estabelecido pelas regras $\mathrm{R} 1$ e $\mathrm{R} 2$ e pelas restrições, que não permite subdividir lotes que gerem outros com faces menor de $20 \mathrm{~m}$ e $15 \mathrm{~m}$. Porém, não é possível determinar - limite de generalidade do método, pois a pouca quantidade de regras possibilita uma vasta opção de variações na inferência das regras a cada passo, como ilustra a figura 6 .

Um conjunto de massas edificadas foi simulado considerando o novo dimensionamento e rearranjo dos lotes (produto da aplicação das regras e restrições à quadra 1). Através da simulação, fica perceptível que alguns dos parâmetros de projeto delimitados pelas leis e códigos de construção municipais, analisados ao criar as regras, foram mantidos e outros desconsiderados. Os parâmetros de recuo em geral e gabarito foram respeitados, porém os que definiam a dimensão da testada dos lotes e a taxa de ocupação foram desconsiderados para que fosse possível visualizar uma

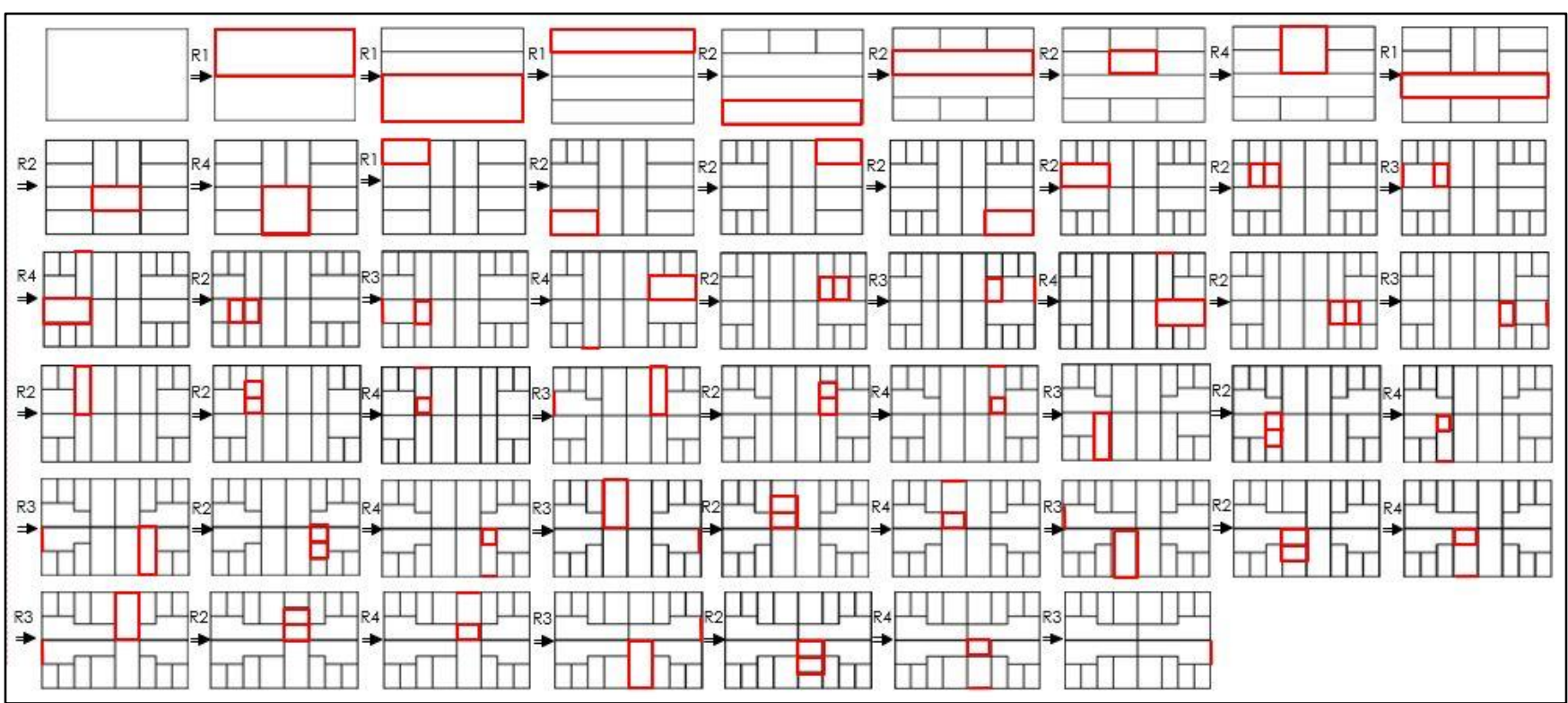

Figura 5: Derivação da quadra 1 com destaque do passo a passo ao aplicar as regras; Fonte: Autores (2018). 


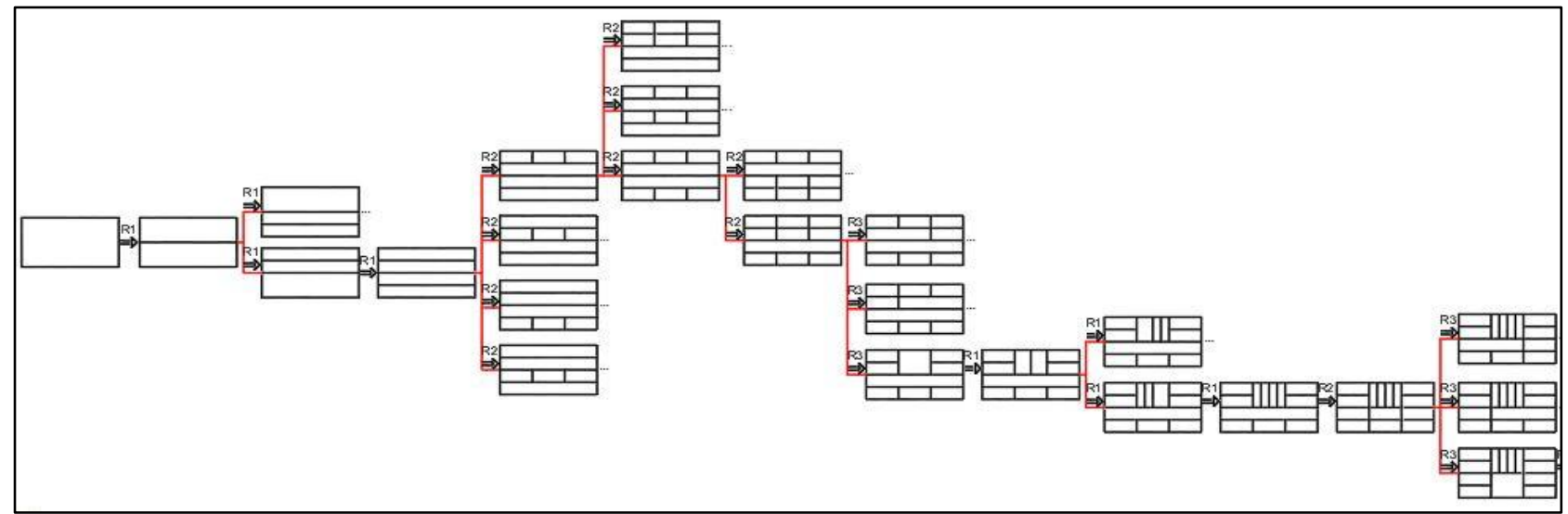

Figura 6: Trecho do processo de derivação da quadra 1 com destaque para as possíveis variações na inferência das regras a cada passo;

Fonte: Autores (2018).

mudança de cenário onde o adensamento aliado à diversidade da forma/volume da edificação fosse evidenciado.

\section{RESULTADOS E DISCUSSÃO}

As derivações realizadas a partir do corpus selecionado mostram quão eficiente se mostrou a gramática da forma para a geração de lotes urbanos. Por meio das regras e restrições inferidas pôde-se desenvolver muitas possibilidades de distribuições de lotes dentro das quadras selecionadas.

É possível observar que a variabilidade dos formatos dos lotes, bem como seus posicionamentos sugerem um número significativo de possibilidades de configurações morfológicas dos lotes nas quadras.

Para ilustrar um dos resultados da derivação por meio das simulações de massas edificadas, poderíamos ter selecionado qualquer uma das derivações durante 0 processo. Entretanto como critério de escolha, decidimos utilizar o cenário da última derivação possível pela aplicação das regras na quadra 1 (ver figura 7).

Para o desenvolvimento das massas, consideramos a legislação municipal, o Código de Urbanismo da cidade de João Pessoa - PB, que classifica os usos permitidos e defini taxa de ocupação, gabaritos e afastamentos das edificações. Desse modo, tanto o processo de derivação dos lotes pela gramática da forma como a simulação de edifícios foram realizados condizentes com a legislação vigente local, mostrando-se um método válido para gerar novas soluções morfológicas.

O critério principal para o estudo de massas foi o de privilegiar as pessoas, criando espaços de conexão e caminhabilidade, organizando as edificações com fluidez e que possibilitasse ao pedestre várias opções de acesso ao interior da quadra. Além desses, outros critérios considerados foram: permeabilidade do solo nas áreas dos lotes e em um grande espaço comum público/privado no interior da quadra; escalonamento de gabaritos permitindo melhor ventilação e iluminação das edificações; possibilidades de usos diferentes como residencial, comercial e de serviços; possibilidade de adensamento populacional justificando e viabilizando investimento em infraestruturas urbanas (BERTAUD, 2004; GLASER, 2012; FARR, 2013; GEHL, 2013; SILVA at. al., 2016).
O resultado obtido da derivação da quadra 1 , onde as regras foram aplicadas o máximo de vezes possível, foi simulado em uma maquete eletrônica 3D, e aponta a gramática da forma como método eficaz em propor novos arranjos de lotes (ver figura 7). No entanto, o processo poderia ter sido interrompido anteriormente, conforme o desejo do projetista, resultando em outra configuração de lotes. O mesmo pode ser feito em relação a simulação dos edifícios, que pode eleger tanto a simulação de
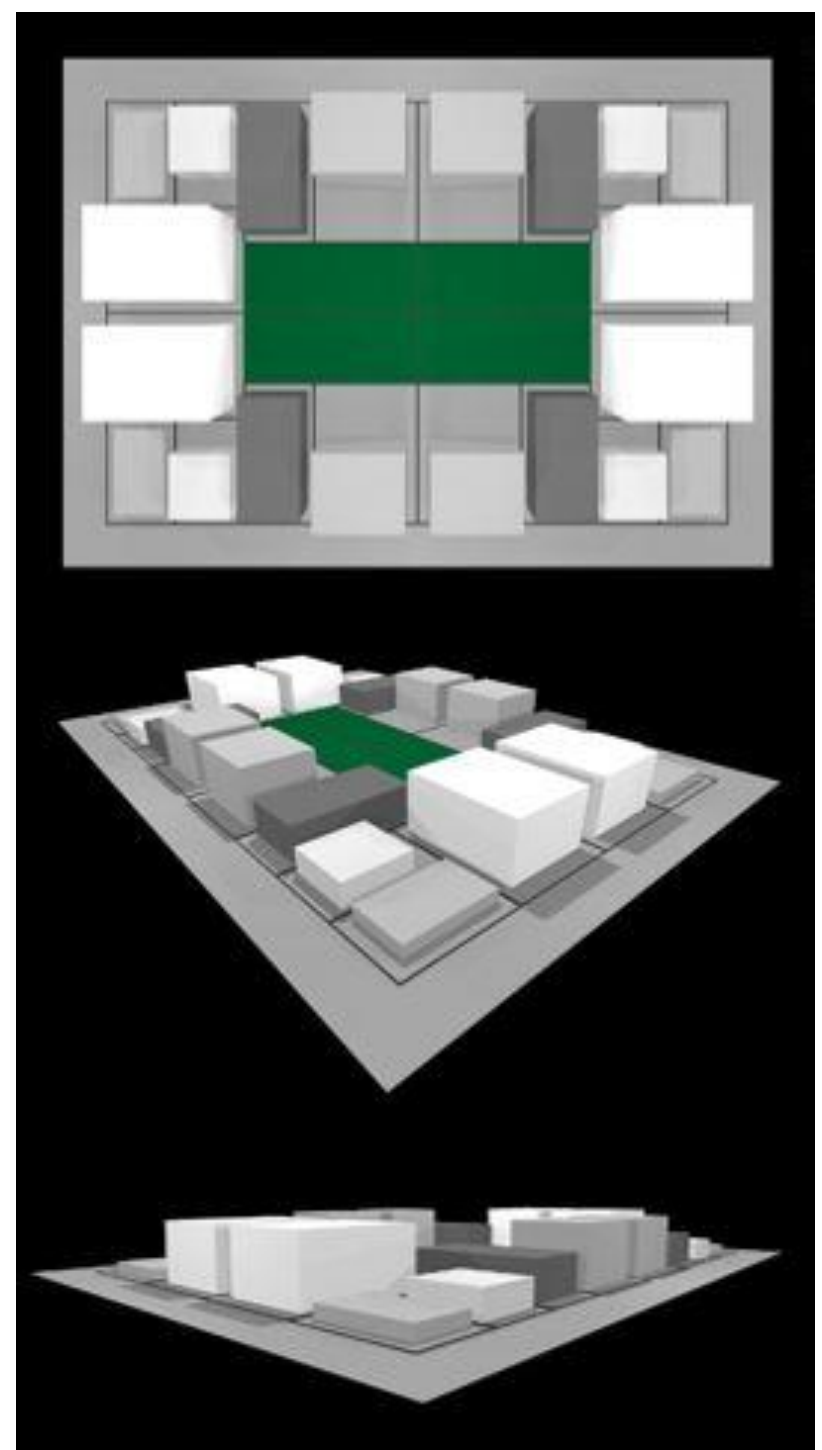

Figura 7: Estudos de massas a partir da quadra 1. Fonte: Autores (2018). 


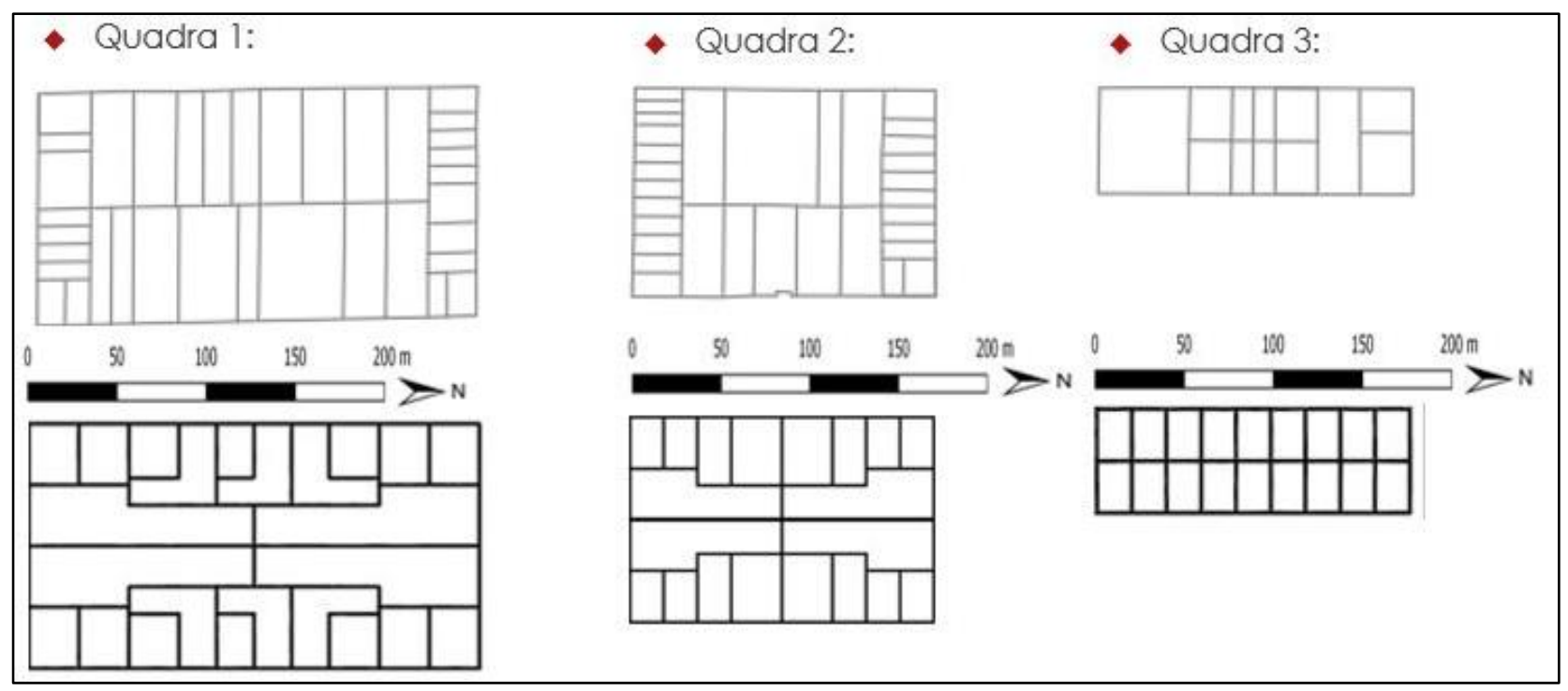

Figura 8: Resultados das derivações das quadras 1, 2 e 3, respectivamente; Fonte: Autores (2018).

edifícios isolados em propriedades privadas, que é em que consiste a maioria da configuração edilícia existe, como poderia eleger a simulação de conjuntos multifuncionais e espaços semipúblicos comuns.

Destaca-se ainda, que, no desenvolvimento dessa gramática, todas as regras e restrições foram aplicadas em todas as quadras sem a necessidade de alteração ou complementação das mesmas. Pode-se considerar que as quadras selecionadas representam as demais quadras do bairro por se assemelharem em dimensionamento e, portanto, a aplicação do método torna-se válida para casos similares.

Ao analisar os resultados obtidos através do processo generativo, conclui-se que o produto das derivações em função das regras e restrições inferidas, podem ou não serem satisfatórios. Isso vai depender se o produto obtido correspondeu aos objetivos lançados na fase inicial do processo e se atende ou não aos critérios elencados. Nesse caso, em que o objetivo é gerar lotes de tamanhos variados para que haja diversidade no arranjo das edificações, lotes com dimensionamento equivalente ao da quadra 3 podem apresentar resultados insatisfatórios. O resultado da derivação da quadra 3 (ver figura 8), pelo fato de possuir uma determinada proporção, mostrou uma distribuição de lotes monótona e trivial. No entanto, é válido ressaltar que todas as regras e restrições puderam ser aplicadas na quadra 3, ainda que o resultado obtido não tenha sido o almejado.

No caso da gramática desenvolvida no presente artigo, os produtos das derivações das quadras 1 e 2 (ver figura 8), foram mais satisfatórios por apresentar maior variação de arranjo dos lotes e possibilidades de configurações espaciais morfológicas diferentes.

Dessa forma, constata-se que em quadras estreitas, mediante o corpus selecionado, as regras e restrições inferidas baseadas na legislação da cidade de João Pessoa - PB, não produzem arranjos internos que corroboram com os critérios e preceitos do urbanismo sustentável ora relatados. Para este formato de quadra não encontramos como resultado, variabilidade nos tamanhos e formas dos lotes, o que não contribui qualitativamente, inviabilizando o uso do método.
A Gramática da Forma mostrou-se eficaz ao ser aplicada no desenvolvimento das pesquisas na busca de soluções e propostas para o desenvolvimento de um urbanismo sustentável. Esta é uma fase inicial da pesquisa e estes são estudos incipientes que apresentam aplicações deste sistema generativo da gramática da forma no processo de investigação para gerar lotes urbanos a partir de um corpus tradicional de um conjunto de quadras no bairro Valentina, em João Pessoa-PB.

\section{REFERÊNCIAS}

BEIRÃO, José Nuno; MENDES, Leticia Teixeira; CELANI, Gabriela. O uso do CIM (city information modeling) para geração de implantação em conjuntos de habitação de interesse social: uma experiência de ensino. Gestão \& Tecnologia de Projetos, v. 10, n. 2, p. 101-112, 2015.

BERGHAUSER PONT, Meta.; HAUPT, Per. (2010). Spacematrix: Space, Density and Urban Form. Rotterdam: NAI Publishers, 2010.

BERTAUD, Alain. The spatial organization of cities: Deliberate outcome or unforeseen consequence?. 2004.

CELANI, Gabriela. An educational experiment with shape grammars and computer applications. International Journal of Design Computing, v. 3, 2002.

CELANI, Gabriela et al. A gramática da forma como metodologia de análise e síntese em arquitetura. Conexão-Comunicação e Cultura, v. 5, n. 10, 2006.

DUARTE, José Pinto. Towards the mass customization of housing: the grammar of Siza's houses at Malagueira. Environment and planning B: Planning and Design, v. 32, n. 3, p. 347-380, 2005.

DUARTE, José Pinto; DE CASTRO, Maria da Conceição Antunes. Personalizar a habitação em série: uma gramática discursiva para as casas da Malagueira do Siza. Fundacion Calouste Gulbenkian, Fundação para a Ciência e a Tecnologia, 2007.

ELOY, Sara et al. Transformation Grammar for Housing Rehabilitation: from a specific to a general grammar. In: Digital Physicality-Proceedings of the 30th eCAADe Conference-Volume 1. Czech Technical University in Prague, 2012. p. $471-478$

FARR, Douglas. Urbanismo Sustentável: desenho urbano com a natureza. Bookman Editora, 2013. 
FISCHER, Thomas; HERR, Christiane M. Teaching generative design. In: 2001): Proceedings of the 4th Conference on Generative Art. 2001.

GAUZIN-MÜLLER, Dominique; FAVET, Nicolas; MAES, Pascale. Arquitectura ecológica: 29 ejemplos europeos. Gustavo Gili, 2002.

GAUZIN-MULLER, Dominique. Arquitetura Ecológica, 304 p. São Paulo. Editora Senac São Paulo, 2011.

GEHL, Jan. Cities for people. Island press, 2013.

GLASER, Meredith (Ed.). The city at eye level: Lessons for street plinths. Eburon Uitgeverij BV, 2012.

JACOBS, Jane. Morte e vida de grandes cidades. Martins Fontes, 2000.

KNIGHT, Terry W. Color grammars: the representation of form and color in designs. Leonardo, p. 117-124, 1993.

KNIGHT, T. W. Shape grammars and color grammars in design. Environment and Planning B: Planning and Design, v. 21, n. 6, p. 705-735, 1994.

RICHARDSON, Roberto Jarry. Pós-Graduação-MetodologiaPesquisa Social: Métodos e Técnicas-Métodos Quantitativos e Qualitativos-Capitulo 5. Editora ATLAS SA-2015-São Paulo, 2017.

ROLNIK, Raquel; CYMBALISTA, Renato; NAKANO, Kazuo. Solo urbano e habitação de interesse social: a questão fundiária na política habitacional e urbana do país. Revista de Direito da ADVOCEF, v. 1, n. 13, p. 123-158, 2011.

SILVA, Geovany Jessé Alexandre da; SILVA, Samira Elias; NOME, Carlos Alejandro. Densidade, dispersão e forma urbana: Dimensões e limites da sustentabilidade habitacional. Arquitextos Vitruvius, n. 189.07, 2016.

STINY, George; GIPS, James. Shape grammars and the generative specification of painting and sculpture. In: IFIP Congress (2). 1971.

STINY, George. Ice-ray: a note on the generation of Chinese lattice designs. Environment and Planning B: Planning and Design, v. 4, n. 1, p. 89-98, 1977.

STINY, George. Introduction to shape and shape grammars. Environment and planning B: planning and design, v. 7, n. 3, p. 343-351, 1980.

STINY, George. Shape: talking about seeing and doing. MIt Press, 2006. 